\title{
Current Control Strategy of Cascaded STATCOM with Compensation of Harmonics and Asymmetric Load
}

\author{
Xuehua Zhao ${ }^{1}$, Liping $\mathrm{Shi}^{1}$ and Zhenglong $\mathrm{Xia}^{2}$ \\ ${ }^{1}$ School of Information and Electrical Engineering, China University of Mining \\ and Technology, Xuzhou 221116, China \\ ${ }^{2}$ School of electrical engineering \& automation, Jiangsu Normal University, \\ Xuzhou 221116, China \\ cumtzxh2013@126.com
}

\begin{abstract}
As the current research focus of the reactive power compensation, the Static Synchronous Compensator (STATCOM a.k.a. SVG) is widely used to realize the compensation of the reactive, harmonic and asymmetric currents. The traditional controller, which is composed of several proportional-integral (PI) controllers, has difficulties to eliminate the steady-state error when the STATCOM needs to provide the compensation ability of the harmonic and asymmetric currents. Therefore, the composite controller composed of the resonant and PI controllers is introduced to realize the tracking control of the DC instruction currents based on the $d-q$ synchronous reference frame. Consequently, the high tracking accuracy and the rapid response character are integrated in the advanced controller. To make the compensation more flexible, a detection approach of the reference harmonic current based on the $d-q$ synchronous reference frame and the discrete Fourier transform (DFT) is proposed and applied to Statcom. The accuracy of the algorithm and frequency applicability of the composite controller is improved by adapting the design of the software phase-locked loop. Moreover, based on the combined circuit topology with multi-field programmable gate array (FPGA), the double-loop control algorithms with the current inner loop and the capacitor voltage outer loop are set up in this paper. The simulation and experiment platform is employed to verify the feasibility and effectiveness of the control strategy.
\end{abstract}

Keywords: Static Synchronous Compensator; Asymmetric current compensation; Harmonic current compensation; Resonant control; Reference current detection; MultiFPGA structure

\section{Introduction}

Large numbers of the Static Synchronous Compensator (STATCOM a.k.a. SVG) is used to regulate dynamic reactive power and solve dynamic voltage stability problems because of its excellent characteristics. The STATCOM is not only used to provide the compensation of the reactive current, but is also adopted to realize the compensation the harmonic and asymmetric currents. At present, with the wide application of non-linear, discontinuous and unbalanced equipment in various kinds of electrical and electronic devices, the problems of three-phase asymmetric load, harmonic suppression and reactive power compensation are research focus in recent years [1-3].

The compensation effect of STATCOM is mainly depends on the performance of the controllers. The reference reactive power and harmonic current instruction signals are obtained by detecting the real-time detection of the load currents and using a special algorithm. After the realization of the command signals, two output current control strategies of STATCOM are used for compensating reactive power and harmonic currents which includes direct-current control and indirect-current control. The indirect-current 
control method is the most common kind of control mode, is structure simple and mature characteristics. But its poor dynamic performance is demonstrated in the application process. Therefore, direct-current control, which has the ability of cancelling harmonic currents and compensating reactive power and three-phase asymmetric loads at the same time, is adopted in this paper [4]. Proportional-integral (PI) is the most common control method used in direct-current control. The static error free tracking control of instruction current, which is DC signal under the $d-q$ synchronous reference frame, is realized when the STATCOM only needs to provide the compensation of the reactive current. The proportional-integral (PI) controller in the system may not perform well in a case where the reference instruction signals of the harmonic and asymmetric currents are AC signals in the $d-q$ synchronous reference frame. In order to realize the detecting method for selective harmonic compensation and the application of proportional-integral (PI) control, multiple synchronous rotating coordinates method is proposed by some literatures to transform selective harmonic currents into DC signals [5, 6]. But during actual designing and operation process, some problems that include introducing too many coordinate transformations with multiple frequencies and accomplishing a group of complex calculation with proportional-integral (PI) controllers may arise. Another solution is to use repetitive controllers [7-10]. The repetitive controllers, which make the output of the system track the periodic reference signals, is applied to take a closed loop control to selected harmonic currents. However, the repetitive controller, which is currently processing the internal model control output on fundamental period, has cycle delay problem.

In order to further simplify the controllers for fundamental and harmonic currents, this paper presents a control algorithm composed of the resonant and PI controllers that compensate the fundamental current and eliminate selective harmonics by adopting $d-q$ synchronous reference frame. The compensation of two kinds of harmonic currents is accomplished at the same time. Therefore, the composite controller could efficiently decrease the number of controllers in the $d-q$ synchronous reference frames. Meanwhile, based on the $d-q$ synchronous reference frame and the discrete Fourier transform (DFT), a new detection approach is used to selectively compensate the $5^{\text {th }}$ and $7^{\text {th }}, 11^{\text {th }}$ and $13^{\text {th }}$, and $17^{\text {th }}$ and $19^{\text {th }}$ harmonic currents of nonlinear load [11-13].

The simulation models and experimental devices used to verify the rationality of the system structure and control strategies are established based on the development background of the medium-voltage cascaded STATCOM. The dynamic and steady-state performances are analyzed in the simulation and experiment. Accordingly, the simulation and experiment results show that the proposed models and strategies are feasible and effective. Moreover, the reactive current of the STATCOM has rapid dynamic response and good stability characteristics.

\section{Main Circuit Structure of Cascaded STATCOM}

The established main circuit model of the medium-voltage cascaded STATCOM is shown in Figure 1. $v_{s a}, v_{s b}$ and $v_{s c}$ denote the three-phase voltage of the power grid; $i_{s a}, i_{s b}$ and $i_{s c}$ are the three-phase current of the power grid; $i_{L a}, i_{L b}$ and $i_{L c}$ represent the three-phase current of the load; $i_{2 a}, i_{2 b}$, and $i_{2 c}$ denote the three-phase output current of the filter; $i_{l a}, i_{l b}$ and $i_{l c}$ are the three-phase output current of the STATCOM; $v_{a}, v_{b}$ and $v_{c}$ represent the three-phase output voltage of the STATCOM. 


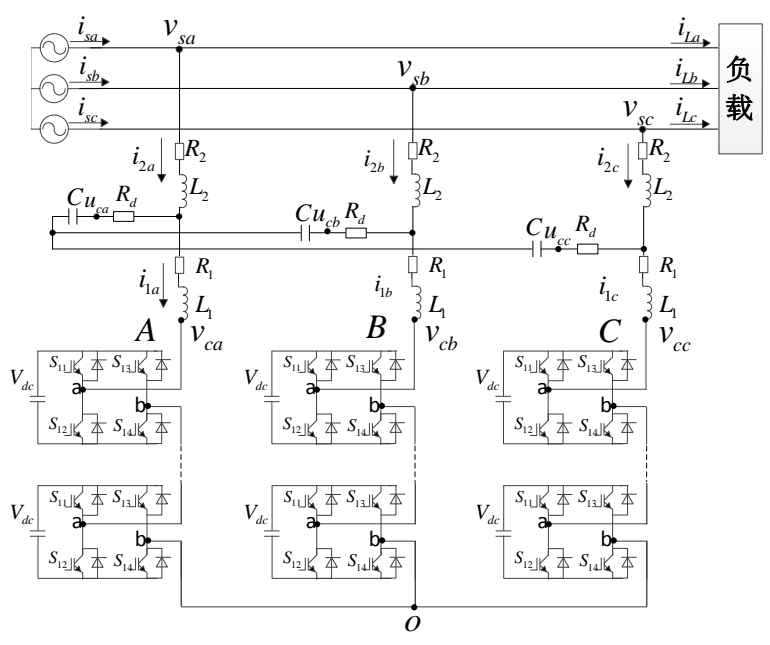

Figure 1. Main Circuit Structure of Cascaded STATCOM

Each phase of the medium-voltage cascaded STATCOM comprises eight H-bridge cells adopting the cascaded structure technology and star connection method. The LCL filter that realizes the optimized control for the output current is adopted to reduce the current harmonics around switching frequency of the three-phase pulse width modulation (PWM) rectifiers.

\section{Current Control Strategies of STATCOM}

\subsection{Tracking Control Strategy of Instruction Current based on PI Control}

Because there is no the zero-sequence component in the three-phase and three-wire system, the load current is expressed as Eq. (1).

$$
\left\{\begin{array}{l}
\left\{i_{a}(n)=I_{1+} \cos \left(\omega t+\theta_{1+}\right)+I_{1-} \cos \left(\omega t+\theta_{1-}\right)+\sum_{n=6 k \pm 1, k=1}^{\infty}\left[I_{\mathrm{n}+} \cos \left(n \omega t+\theta_{n+}\right)+I_{\mathrm{n}-} \cos \left(n \omega t+\theta_{n-}\right)\right]\right. \\
i_{b}(n)=I_{1+} \cos \left(\omega t+\theta_{1+}-\frac{2 \pi}{3}\right)+I_{1-} \cos \left(\omega t+\theta_{1-}+\frac{2 \pi}{3}\right)+\sum_{n=6 k \pm 1, k=1}^{\infty}\left[I_{\mathrm{n}+} \cos \left(n \omega t+\theta_{n+}-\frac{2 \pi}{3}\right)+I_{\mathrm{n}-} \cos \left(n \omega t+\theta_{n-}+\frac{2 \pi}{3}\right)\right] \\
i_{c}(n)=I_{1+} \cos \left(\omega t+\theta_{1+}+\frac{2 \pi}{3}\right)+I_{1-} \cos \left(\omega t+\theta_{1-}-\frac{2 \pi}{3}\right)+\sum_{n=6 k \pm 1, k=1}^{\infty}\left[I_{\mathrm{n}+} \cos \left(n \omega t+\theta_{n+}+\frac{2 \pi}{3}\right)+I_{\mathrm{n}-} \cos \left(n \omega t+\theta_{n-}-\frac{2 \pi}{3}\right)\right]
\end{array}\right.
$$

The signals (i.e., $I_{\mathrm{n}+}$ and $I_{\mathrm{n}-}$ ) are the amplitudes of the positive sequence and negative sequence components in the $\mathrm{n}$ times the harmonic current. The three-phase load currents (i.e., Eq. (1)) are expressed as follows using the $d-q$ synchronous reference frame.

$$
\left\{\begin{array}{l}
\left\{i_{d}(n)=\sqrt{\frac{3}{2}}\left\{\left[I_{1+} \cos \left(\theta_{1+}\right)+I_{1-} \cos \left(2 \omega t+\theta_{1-}\right)\right]+\sum_{n=6 k, k=1}^{\infty}\left\{I_{(\mathrm{n}+1)+} \cos \left[n \omega t+\theta_{(n+1)+}\right]+I_{(\mathrm{n}-1)-} \cos \left[n \omega t+\theta_{(\mathrm{n}-1)-}\right]\right\}\right\}\right. \\
i_{q}(n)=\sqrt{\frac{3}{2}}\left\{\left[I_{1+} \sin \left(\theta_{1+}\right)-I_{1-} \sin \left(2 \omega t+\theta_{1-}\right)\right]+\sum_{n=6 k, k=1}^{\infty}\left\{I_{(\mathrm{n}+1)+} \sin \left[n \omega t+\theta_{(n+1)-}\right]-I_{(\mathrm{n}-1)-} \sin \left[n \omega t+\theta_{(\mathrm{n}-1)-}\right]\right\}\right\}
\end{array}\right.
$$

The above results shows that the degree of the positive sequence component is reduced by once and the negative sequence components degree is increased by once. Subsequently, the fundamental positive sequence component is transformed into DC signal in $d-q$ coordinates. Therefore, while the STATCOM is only used to compensate the fundamental reactive current component, the instruction current is DC signal and PI controllers are used to realize static error free tracking control of the instruction current under the two-phase static coordinate. 


\subsection{Selective Current Control Strategy using Composite Controller}

The AC signal is included in the instruction current when the STATCOM needs to provide the compensation ability of the harmonic and asymmetric currents. In the three-phase and three-wire system, the $(6 n+1)$ times the characteristic harmonic components are the main compositions of the positive sequence. Meanwhile, the negative sequence components are consists mainly of the $(6 n-1)$ times the characteristic harmonic currents [9]. The Eq. (2) shows that the $(6 n \pm 1)$ times the harmonic currents of the positive and negative sequence parts in the $a-b-c$ coordinates are then transformed into the $6 n$ times the control part in the $d-q$ coordinates. Moreover, there is the $2^{\text {th }}$ harmonic component of the fundamental negative sequence under the three phase unbalanced loads. Accordingly, the proportional-integral (PI) cannot be applied to the control of the DC signal of the fundamental current, the 2 and $6 n$ times the harmonic currents included in the instruction currents with zero steady-state error when the STATCOM needs to provide the comprehensive compensation. Therefore, a new control strategies including the proportional-integral (PI) and resonant control technologies are presented in this paper by introducing the resonant controllers in the $d-q$ synchronous reference frame. The tracking control of the $(6 n \pm 1)$ times the harmonic currents in the instruction currents in the $a-b-c$ coordinates is then transformed into the realization of the $6^{\text {th }}, 12^{\text {th }}$, and $18^{\text {th }}$ harmonic currents control in the $d-q$ coordinates. In this paper, the proposed strategy is used to simplify the design of the controllers effectively, and the number of the controllers will be reduced by half. The block diagram of the composite controller is shown in Figure 2.

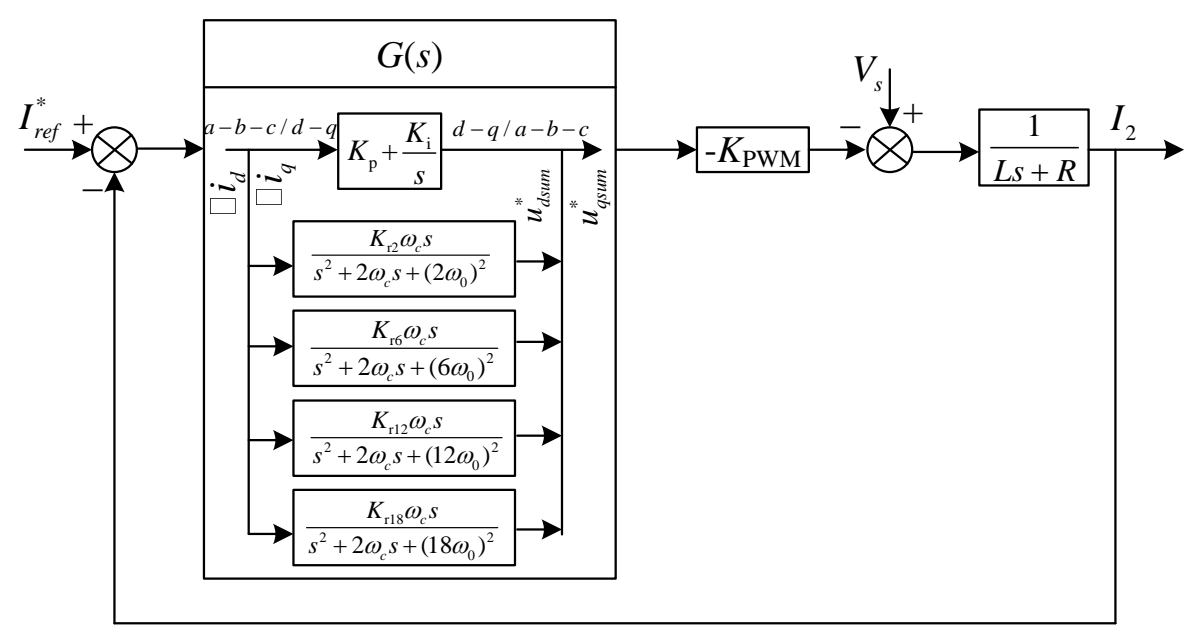

Figure 2. Block Diagram of Composite Controller

The open-loop amplitude frequency characteristics of the compound controller are analysed (Figure 3 (a)). And its closed-loop amplitude frequency characteristics are shown in Figure 3 (b). As the above results show, the compound controller has infinite gain for the selective harmonic currents in the resonance frequencies (i.e., $\omega_{n}=6 n \omega_{0}$ ). On the contrary, the gain loss is significant where the frequency deviates from the resonance point. Accordingly, the compound controller is chosen to realize the accurate compensation of the selective harmonic currents with zero steady-state error for the STATCOM system. 


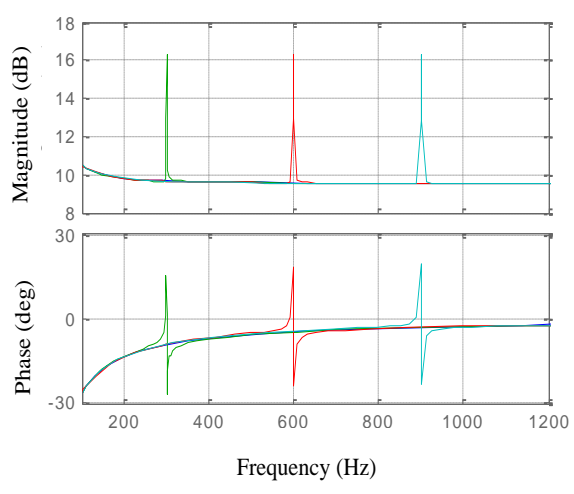

(a) Open-loop Characteristics

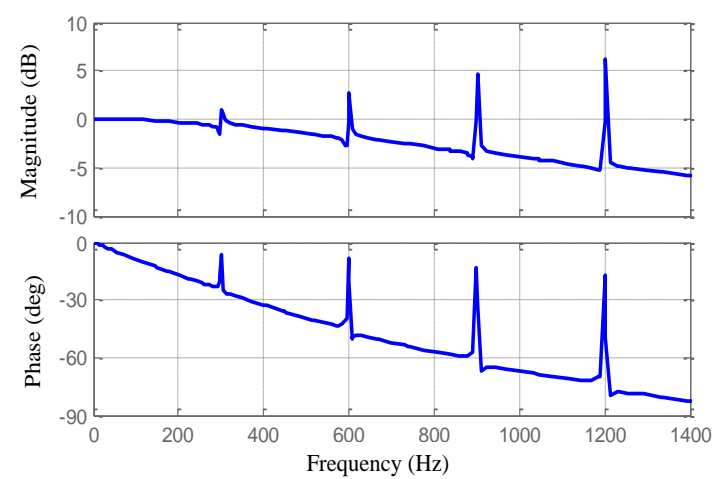

(b) Closed-loop Characteristics

\section{Figure 3. Open-loop and Closed-loop Characteristics of Compound Controller}

\subsection{New Detection Approach of Harmonic Currents based on $d-q$ Synchronous Reference Frame and Discrete Fourier transform (DFT)}

The output frequency bandwidth, which has the control ability of canceling harmonic and asymmetric currents while compensating the reactive power, is finite. The high frequency harmonic currents of STATCOM may not only fail to cancel the harmonic currents, but instead could amplify the corresponding harmonic currents. Consequently, this paper proposes a more flexible detection approach for selective harmonic currents based on the $d-q$ synchronous reference frame and discrete Fourier transform (DFT). The $2^{\text {th }}$ and $6 n$ times the harmonic currents included in the instruction current are derived after the further processing of the instruction currents (i.e., $i_{d}$ and $i_{q}$ ) with the coordinate transformation and discrete Fourier transform (DFT) algorithms.

The periodic signal with harmonic currents is defined as $X(t)$, where the variables $T$ and $\omega$ represent the corresponding signal cycle and angular frequency, respectively. From the sampled data of $X(t)$, the corresponding expression form based on DFT algorithm is presented as:

$$
\begin{gathered}
X_{n}(k \tau)=A_{n} \cos (n \omega k \tau)+B_{n} \sin (n \omega k \tau), k=0,1, \ldots, N-1 \\
A_{n}=\frac{2}{N} \sum_{m=0}^{N-1}[X(m \tau) \cos (n \omega m \tau)] \\
B_{n}=\frac{2}{N} \sum_{m=0}^{N-1}[X(m \tau) \sin (n \omega m \tau)]
\end{gathered}
$$

Accordingly, $N$ is the sampling number of the each period and the variable $\tau$ is sampling period. The real part and imaginary part coefficient of harmonic currents with the discrete Fourier transform (DFT) algorithms are transformed into the following form.

$$
\begin{aligned}
& A_{n}(i)=A_{n}(i-1)+\frac{2}{N}[X(i \tau)-X((i-N) \tau)] \cos (n \omega i \tau) \\
& B_{n}(i)=B_{n}(i-1)+\frac{2}{N}[X(i \tau)-X((i-N) \tau)] \sin (n \omega i \tau)
\end{aligned}
$$

The above formulas are established according to the sampling period and the sampled data is done continuously and updated in each sampling period. The above results show that the iterative optimal algorithms not only do not need to calculate the sampling values in the whole sampling period, but also reduce the calculation amount. Subsequently, Eq. 
(6) and Eq. (7) meet the $X(i \tau)=X((i-N) \tau)$ condition and changeless results when the system is in a steady state. Another state is that the system keeps up accurate calculation results after a sampling period delay when the loads are changed. Based on $d-q$ transformation and DFT, the $d$-axis reference current detection method, especially the currents within the $20^{\text {th }}$ the characteristic harmonics, is shown in Figure 4. Accordingly, $q$-axis current detection method, which adopts same current control mode, can be realized by same way.

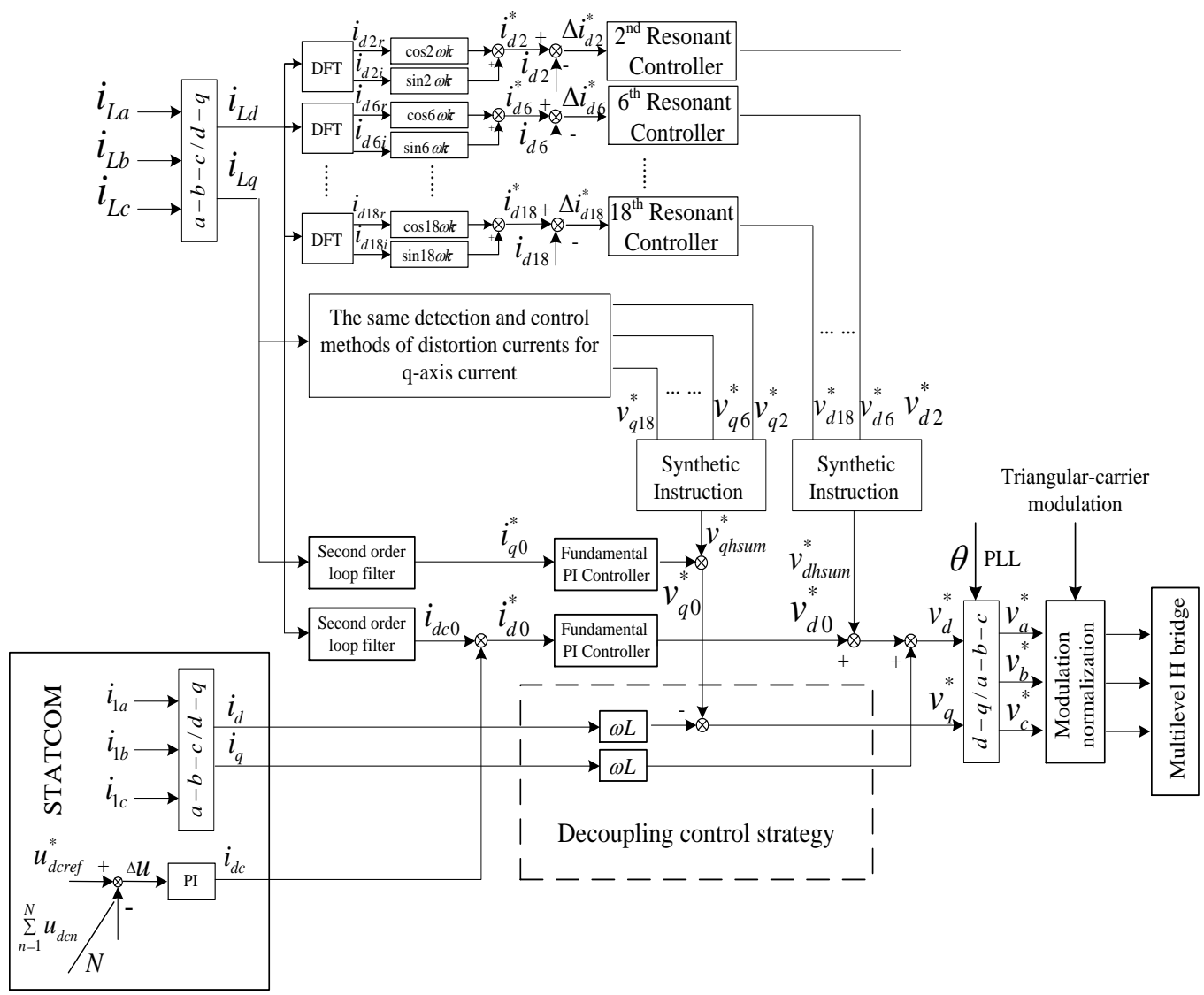

Figure 4. Reference Current Detection Method based on $d-q$ Transformation and DFT

Where, $V_{d c r e f}^{*}$ and $\sum_{n=1}^{N} V_{d c n} / N$ represent the reference value and the real-time average value of the capacitance voltage, respectively. The variables $i_{d c}$ and $i_{d}^{*}$ denote the output of voltage control loop and $d$-axis current reference, respectively. $i_{d n r}, i_{d n i}, i_{q n r}$ and $i_{q n i}$ represent the coefficient of the harmonic currents in the $d-q$ synchronous reference frame (i.e., the $2^{\text {th }}$ and $6 n$ times the harmonic currents).

\subsection{Phase-Locked Loop (PLL) with Fixed Number of Sampling Points}

The above formula is sampled synchronously with the periodic signals of the fundamental positive sequence component. Therefore, in this paper, the detection method with fixed number of sampling points, which should satisfy the following condition in a fundamental period, is proposed to ensure the detection accuracy otherwise the spectrum leakage and fence effects are inevitable to cause the problem of big errors in harmonic detection. 


$$
T=T_{0} / N
$$

Where, $N$ and $T$ represent the sampling number and sampling time, respectively. $T_{0}$ is the time period of fundamental signals in network.

Based on the transformation of synchronous coordinate transformation and software phase-locked loop (SPLL), this paper proposes that real-time adjustment of sampling period can be realized by tracking the fundamental period. when the number of sampling points remains unchanged in a fundamental period, the phase relation between sampling points is determined, such as the default value is $N$ and phase difference is $2 \pi / N$. Accordingly, the variables sin $\omega t$ and $\cos \omega t$ needed in the process of synchronous rotating coordinate transformation are obtained through the analysis of the look-up table method. The phase-locked loop (PLL) design with fixed number of sampling points is as follows.

Firstly, the value of $\sin \omega t$ and $\cos \omega t$ is obtained through the look-up table method, and then the system adopts $d-q$ synchronous reference frame to make the voltage of three phase network to the variables in the $d-q$ coordinates (i.e., $v_{d}$ and $v_{q}$ ). When the variable $\omega t$ has the same value as the voltage phase of A-phase under the balanced three-phase voltage condition, the transformed $q$-axis component should be zero. Therefore, the PI controller, which has the ability of close correcting function to the error between the transformed $q$-axis voltage and zero reference value, is used to realize the phase angle adjustment. On this basis, a new sampling period that is obtained by the above results and the way to construct the next SPLL calculation is given to make the current value of the variable $\omega t$ gradually closer to the voltage phase of A-phase. Figure 5 shows the block diagram of phase-locked loop (PLL) with fixed sampling number.

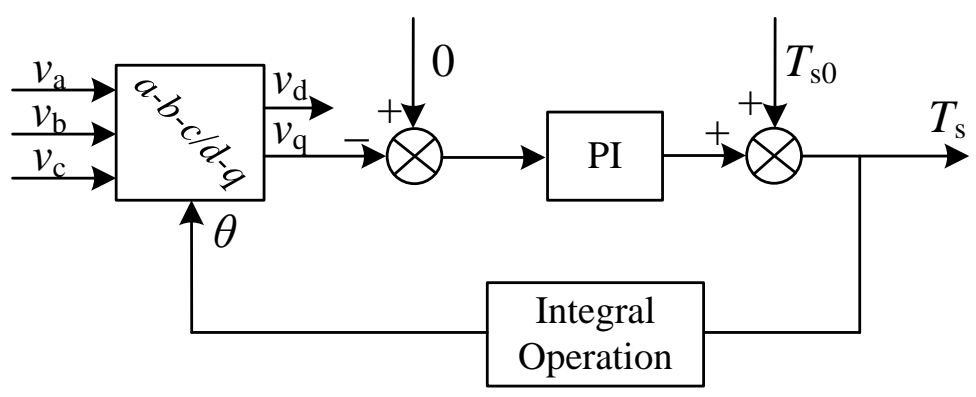

Figure 5. PLL with Fixed Number of Sampling Points

\section{Simulation of Control Algorithm and Structure}

\subsection{Simulation Platform of System}

The system simulation platform based on the main circuit model of the $6 \mathrm{kV}$ STATCOM (Figure 1) is proposed and simulated by MATLAB. The simulation parameters of the STATCOM are shown in Table 1. Because of the fluctuation of power system and the coupling of mathematical model in $d-q$ coordinate system, the paper adopts a current feed-forward decoupling control method. The DC bus voltage and the current control are regulated by the double-loop control algorithms with the current inner loop and the capacitor voltage outer loop. Then, the controller is designed based on the composite controller. The control principle diagram of the $6 \mathrm{kV}$ STATCOM in the simulation process is shown in Figure 6. 
Table 1. Simulation Parameters

\begin{tabular}{c|c}
\hline Parameters & Value \\
\hline System Structure & Cascaded Technology and Star Connection \\
& Method \\
Number of Power Units & 8 \\
DC-side Capacitors & $3000 \mu \mathrm{F}$ \\
Voltage Reference of Capacitors & $750 \mathrm{~V}$ \\
Reactor & $6 \mathrm{mH}$ \\
Sampling Frequency & $10.8 \mathrm{~K}$ \\
Switching Frequency & $5.4 \mathrm{~K}$ \\
Equivalent Resistance of Reactors & $0.6 \Omega$ \\
\hline
\end{tabular}

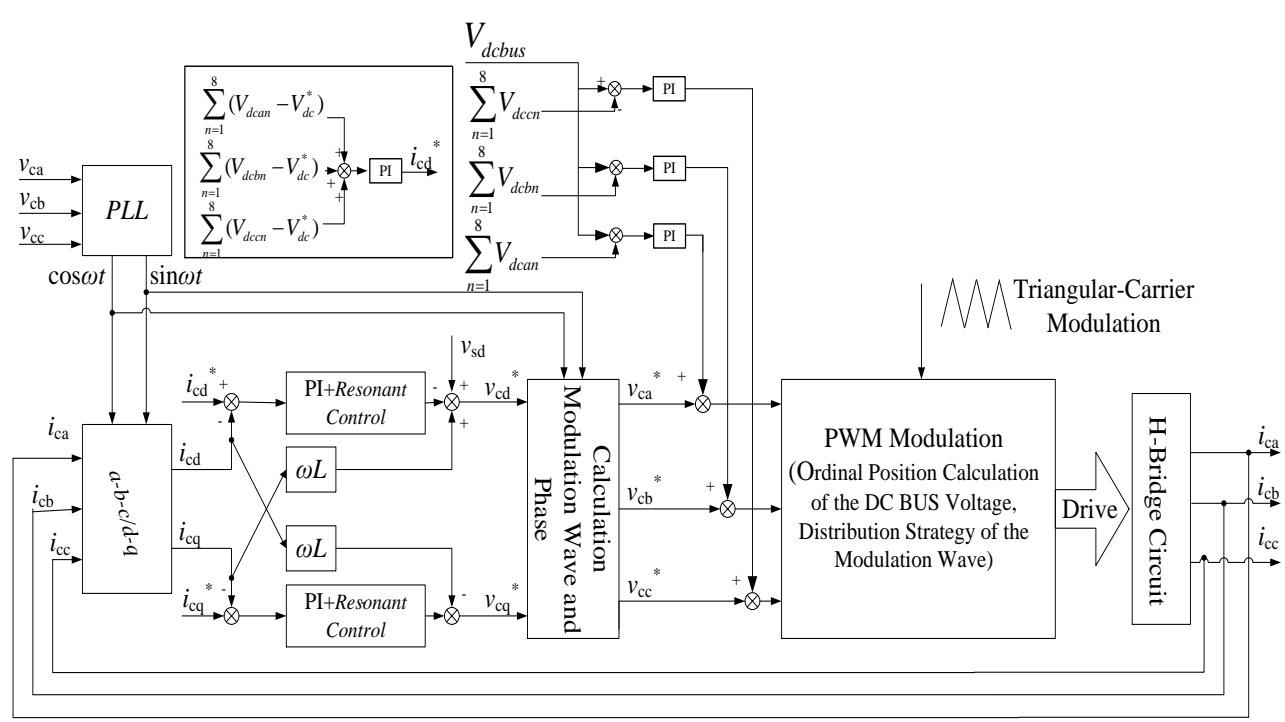

Figure 6. Control Diagram of Cascaded STATCOM

The variable $V_{d c \text { bus }}$ in the preceding model is used as the reference of the phase-to-phase voltages, which should satisfy the following condition: $V_{d c b u s}=\left(\sum_{n=1}^{8} V_{d c a n}+\sum_{n=1}^{8} V_{d c b n}+\sum_{n=1}^{8} V_{d c c n}\right) / 3$. The variables $i_{c d}^{*}$ and $i_{c q}^{*}$ represent the reference of the active and reactive currents, respectively. The actual active and reactive currents $i_{c d}$ and $i_{c q}$, which are transformed from the output currents of STATCOM (i.e., $i_{c a}, i_{c b}$ and $i_{c c}$ ) with the $d-q$ synchronous reference frame, is comparing with $i_{c d}^{*}$ and $i_{c q}^{*}$. The reference voltages (i.e., $v_{c d}^{*}$ and $v_{c q}^{*}$ ) are utilized to achieve the output modulation control using the preceding decoupling method and PI controllers. The three-phase voltage signals generated with triangle and modulation wave signals (i.e., $v_{c a}^{*}$, $v_{c b}^{*}$, and $v_{c c}^{*}$ ) from the main controller are used to drive the H-bridge circuits.

\subsection{Balance Control Scheme of Capacitor Voltage using Unipolar Phase Disposition Modulation and Modulation Wave Distribution Strategies}

The unipolar phase disposition modulation and modulation wave distribution strategies employed as the voltage balance control scheme are investigated to solve the voltage 
unbalance problem of the DC-side capacitor voltage. These strategies are then added to the adaptive dynamic layered control scheme to form the control strategies of the integrated voltage, phase-to-phase voltage, and each unit voltage.

The energy balance principle of the H-bridge structure shows that the DC-side capacitors are always in the charging state when the current is in the same direction as the capacitor voltage. The capacitor absorbs energy through H-bridge. On contrast, the capacitor releases energy, and the capacitor voltage drops (Figure 7). Moreover, the voltage of the capacitance which is on the DC side of the H-bridge is stabilized by charge-discharge balance control in a half-cycle of the fundamental (i.e., whether the areas of the shape 1 and 2 are equal). Therefore, this paper uses the modulation wave distribution methods to solve the voltage unbalance problem of the DC-side capacitors by controlling the insulated-gate bipolar transistor (IGBT) switch frequency and conducting time for the different H-bridges. The specific allocation strategy is as follows.

Firstly, according to the real-time value of the capacitors voltage, the ordinal position of each capacitor voltage is sorted, and then added into an array list. The next step is to implement the distribution of the modulation. When the distribution modulation waves assigned to different H-bridges are not the same, it causes some problems, such as the different charging and discharging active status, different loss allocation and voltage unbalance. Therefore, according to the voltage sorted result of the capacitors, a new algorithm of automatically controlling the voltage balance through the modulation wave distribution strategy is proposed.in this paper. The voltage comparison between the capacitors in the control program is conducted one time per control cycle. The block diagram of modulation allocation is shown in Figure 8.

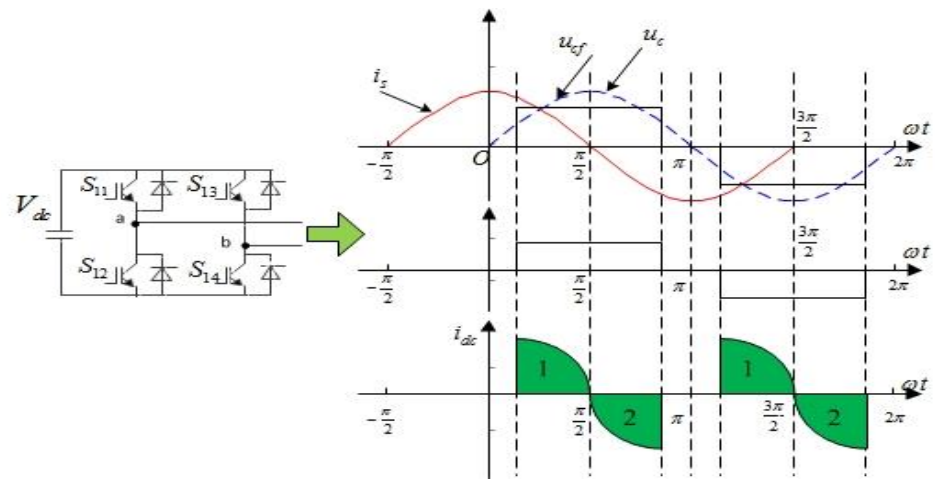

Figure 7. Principle of Charging and Discharging of Capacitors

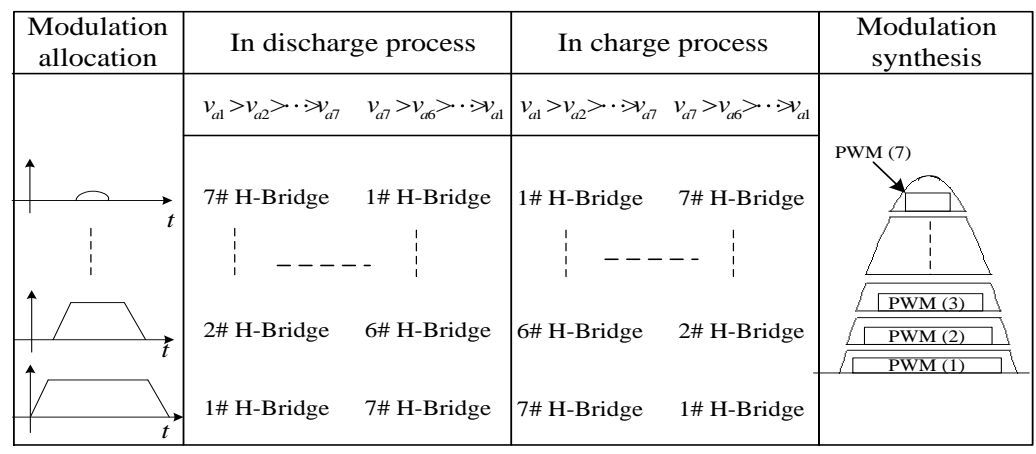

Figure 8. Principle of Modulation Allocation

\subsection{Simulation Analysis}

In this paper, the proposed current control strategy, used as the efficient control scheme of the harmonic and asymmetric currents, is analyzed precisely from three stages. The 
simulation process is as follows.

(1) 0.5 seconds before, the inductive current is used to simulate the load current and its value is $100 \mathrm{~A}$.

(2) 0.5 seconds later, the rectifier load included in the load current is used as the harmonic source model.

(3) at 0.6 seconds, let a resistor connected between A and C phases make as an asymmetric component.

Meanwhile, in order to solve the problem of imbalance reactive load compensation, the asymmetric load model that comprises three-phase unbalanced reactive and rectifier load in the simulation process is also used to simulate actual three-phase unbalanced reactive load reactive power compensation. The use time of the rectifier load is 1.0 seconds.

The current PI regulator parameters are designed using the left half plane zero-pole cancellation method. The parameter settings are as follows. $k_{p}=20, k_{i}=2000$. The sampling frequency adopted in this paper is $10.8 \mathrm{kHz}$, and $N=$ sampling frequency $/ 50=216$. The cut-off frequency of the second-order low-pass filter $S(z)$ should not be less than the $20^{\text {th }}$ harmonic frequency when the STATCOM needs to provide the minimum compensation ability of the $20^{\text {th }}$ harmonic current. In this paper, the frequency of the output filter can be set $2.2 \mathrm{kHz}$ to ensure the bandwidth of control system. And the second-order low-pass filter transfer function is as follows.

$$
S(z)=\frac{0.23977 z^{2}+0.47954 z+0.23977}{z^{2}-0.22115 z+0.18023}
$$

The simulation of the composite controller has also been done to compare the property of the system with that of traditional PI controller. The dynamic performance of the two types of controller is shown in Figures 9-13.

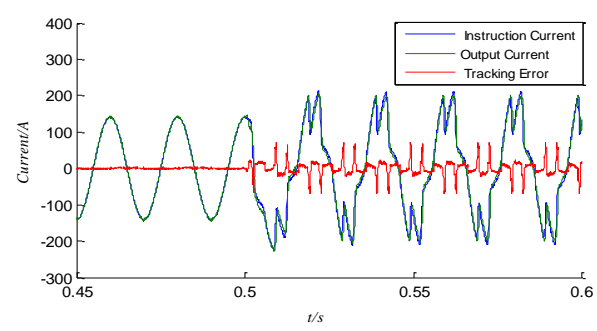

(a) PI Control Waves

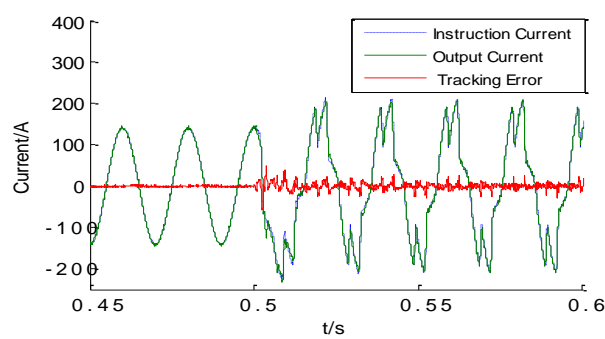

(b) Composite Control Waves

\section{Figure 9. Reference Current, Output Current and Tracking Error of STATCOM}

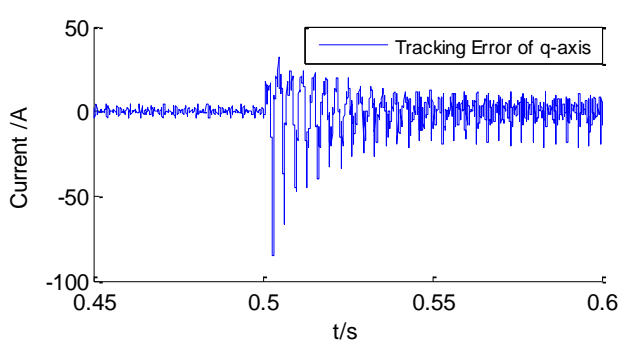

(a) Current Tracking Error Wave in $q$-axis

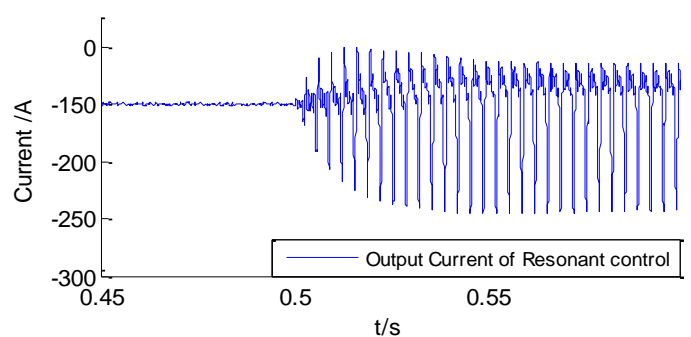

(b) Current Output Wave of Resonant Control

Figure 10. Reference Current Error and Output Signal of Composite Controller in $q$-axis 


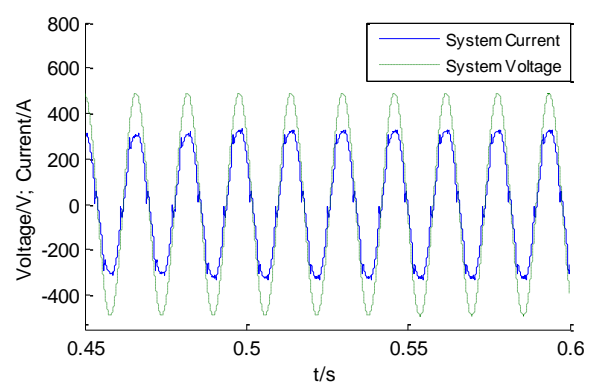

(a) Voltage and Current of PI Control

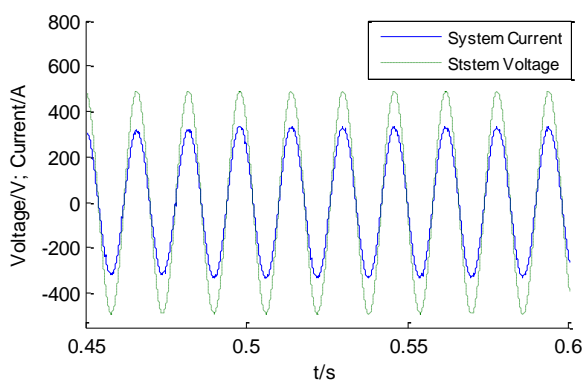

(b) Voltage and Current of Composite Control

Figure 11. System Current and Voltage Waves of STATCOM

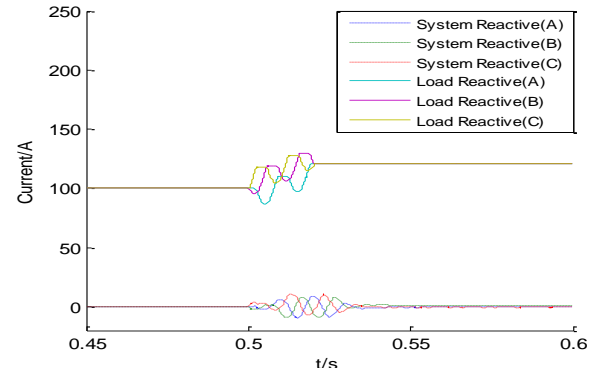

(a) Reactive Currents of System and Load

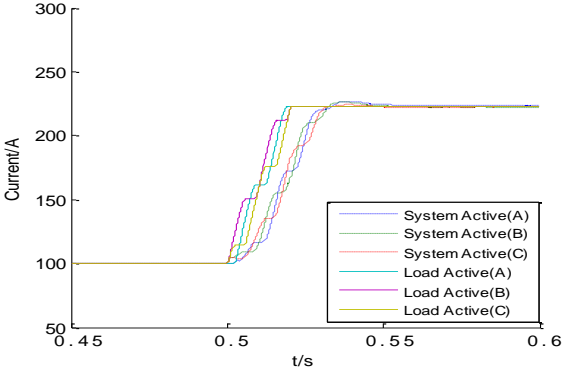

(b) Active Currents of System and Load

Figure 12. Active and Reactive Current when using Composite Controller

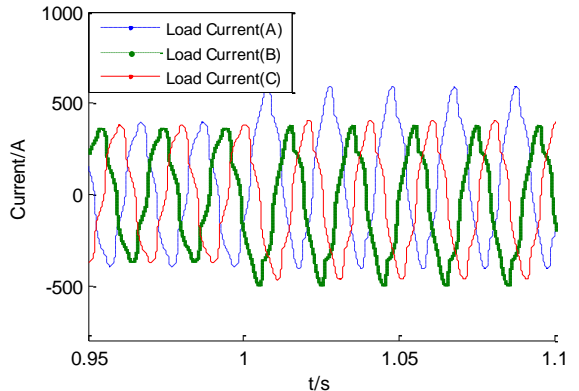

(a) Three Unbalanced Load Current

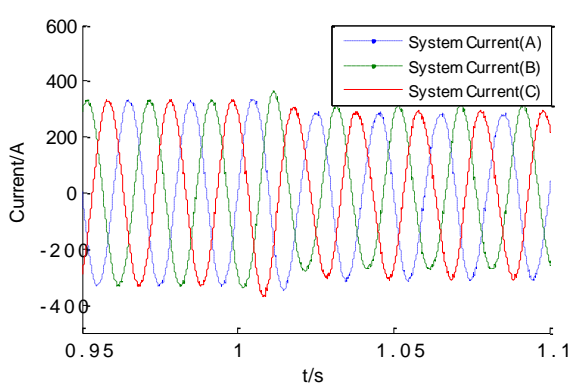

(b) Three Balanced System Current

Figure 13. Three-phase Load and System Currents of Unbalance Loads

The current waves of the reference current, output current and tracking error of the two types of controller are shown in Figures 9. Simulations indicate that the two types of control methods have good control effectiveness when the STATCOM only needs to compensate fundamental current (i.e., 0.6 seconds before). But the harmonic tracking control results shows that the composite controller, which could solve the periodic signal control problem, provides a better control quality, speediness, smaller tracking error than the general PI control system.

The error signal of the $q$-axis tracking control current and output current signal of the resonant controller are analyzed in detail to further verify the control effect of the composite controller. Its waves are shown in Figures 10. At 0.6 seconds before, PI control is the main algorithm for realizing static error free tracking control of the fundamental component transformed into the DC part under two-phase static coordinate, but when there are harmonic currents included in the instruction, traditional PI controller will have higher control error. Moreover, Figures 10 shows that the maximum amplitude of the $q$-axis output current becomes larger and larger as the $q$-axis tracking control error decreases. At about 0.6 seconds, the resonant controller begin to have an impact and the 
output of STATCOM come to steady state after period delay.

As shown in Figures 11, the system voltage and current waves was analysed through two groups of waves collected via two kinds of control strategies. The simulation results indicate that the composite controller can reduce the total harmonic distortion (THD) of the harmonic current from $14.71 \%$ to $2.25 \%$ and suppress the harmonic better relative to PI control.

The RMS current analysis of the active and reactive components is shown in Figures 12. The analysis result shows that the STATCOM achieves dynamic current compensation without the need from the grid. The results in Figures 13 show that the STATCOM provides the excellent compensation results of three-phase asymmetric load, especially the2nd-order harmonic.

\section{Experiment Verification}

\subsection{Experimental Platform of System}

An experimental system based on the main circuit structure of the cascaded STATCOM (Figure 1), multi-field programmable gate array (FPGA) and unipolar phase disposition modulation is developed, and experimental research is conducted. The compensation experiment device (i.e., STATCOM) mainly comprises a control cabinet, several power cabinets, a starting cabinet, and a set of reactors. The high speed FPGA chip, which is produced by Xilinx, and the model is known as XC3SD1800A FG676, has been chosen as the core controller.

The hardware structure of the control system includes FPGA control circuit board, A/D circuit boards, I/O circuit boards, optical fiber interface circuit boards, and power circuit boards using multiple FPGA chips. The automatic control system of the programs in core FPGA realizes the functions of sampling, design of phase-locked loop (PLL), ordinal position calculation of the DC-side voltage, distribution strategy of the modulation wave, operation of double-loop control algorithms and human-machine interface. The integral control principle diagram is shown in Figure 14.

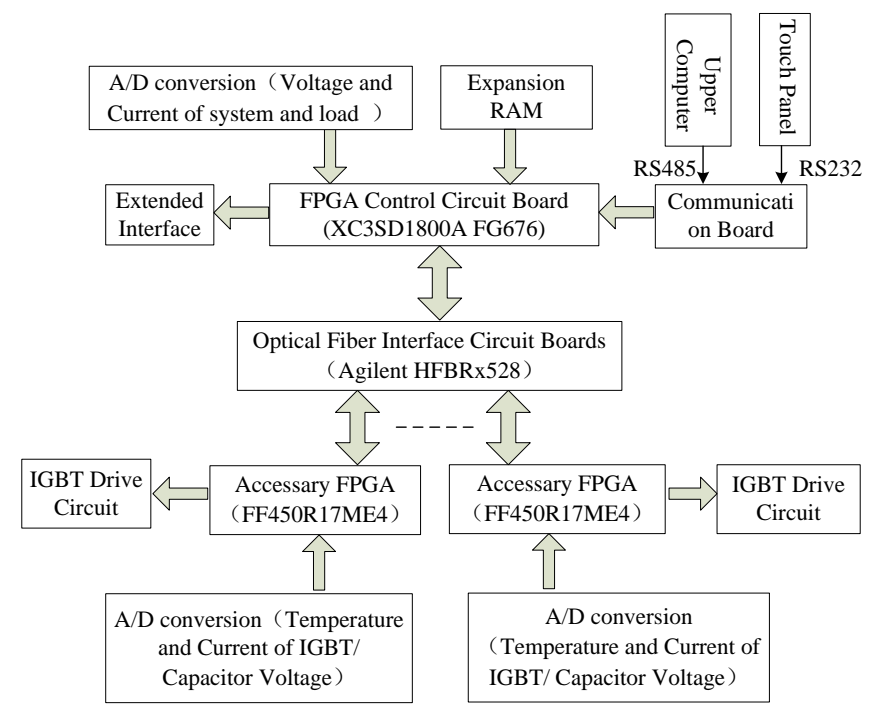

Figure 14. Structure of Multi-FPGA Control

\subsection{Experiment Analysis}

During the experiments, two groups of experimental devices have been developed. One is used to generate reactive and harmonic currents, and another is compensation device. The control strategies proposed in the paper is applied to implement the close-loop control 
for the reactive, harmonic and asymmetric currents compensation and its relevant experimental waveforms are presented. In order to verify the dynamic and static performance, the system has been adopted with alternate varied periodic current. The specific value is 150 (A) and 50 (A). Moreover, the no-sinusoidal current, which contains harmonic components, is generated to further verify the compensation effect of the harmonic currents. The load current THD is $12.01 \%$.

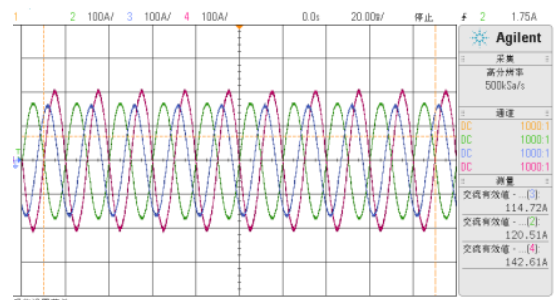

(a) Steady-state Performance

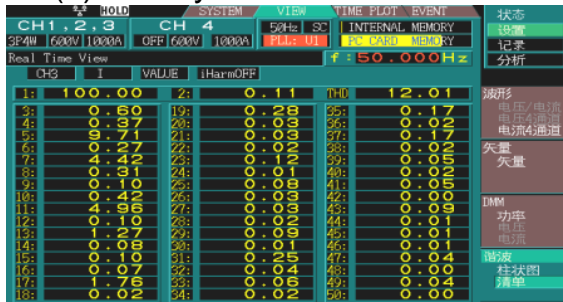

(c) Load Current Components

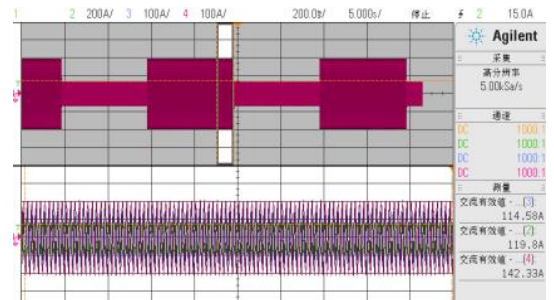

(b) Dynamic Performance

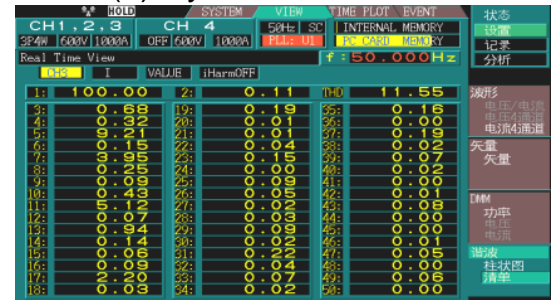

(d) Compensation Current Components

\section{Figure 15. Harmonic Components of Load and Compensation Currents}

As shown in Figure. 15 (a) and 15 (b), the results show that the experimental device can offer better compensation accuracy in steady-state or dynamic performance. The main harmonic components of the load and compensation currents are analyzed in detail (Figure 15 (a) and 15 (b)). The Figure 15 (c) shows that the load current includes not only the fundamental current but also specific higher-order harmonics, such as the $5^{\text {th }}$ and $7^{\text {th }}$, $11^{\text {th }}$ and $13^{\text {th }}$, and $17^{\text {th }}$ and $19^{\text {th }}$ harmonic currents. The main harmonic components of the experimental device current shown in 15 (d) indicate that the frequency spectrum characteristic of compensation current is accordance to the load current.

\section{Conclusion}

In this paper, a new STATCOM control strategy, employed as the efficient control scheme of the fundamental, harmonic and asymmetric currents, has been analyzed in detail from three aspects. Because of the control problem on the compensation of harmonic and asymmetric currents, the composite controller proposed in the paper is introduced to get good compensation performance. Then, a new detection approach of characteristic harmonics, based on the $d-q$ synchronous reference frame and DFT, has been proposed and applied to the STATCOM. Meanwhile, the Phase-locked loop (PLL) with fixed number of sampling points is proposed to ensure the detection accuracy. Both the simulation and experimental results indicate that the comprehensive compensation current has a good dynamic, and steady-state, performance. Therefore, this control strategy should be of great value in engineering applications.

\section{Acknowledgements}

Project supported by the Specialized Research Fund for the Doctoral Program of Higher Education of China (Grant No. 20110095110014). 


\section{References}

[1] B. Singh, R. Saha and A. Chandra, "Static synchronous compensators (STATCOM): a review. Power Electronics”, vol.2, no.4, (2009), pp.297-324.

[2] G. Yuanbo, Z. Xin and Z. Xiaohua, "Nonlinear control of STATCOM under unbalanced condition", Electric Power Automation Equipment, vol.32, no.2, (2012), pp.50-55.

[3] Z. Xianghua, C. Yuanhua and L. Wenhua, "New topology for unbalance compensation of cascaded STATCOM with star configuration", Electric Power Automation Equipment, vol.34, no.2, (2014), pp.108-113.

[4] T. Yu, S. Choi and H. Kim, "Indirect current control algorithm for utility interactive inverters for seamless transfer”, Proc. of PESC’06, (2006); Jeju, Korea.

[5] Z. Shuquan, D. Ke and X. Bin, "Selective harmonic current control based on multiple synchronous rotating coordinates", Proceedings of the CSEE, (2010).

[6] W. Yue, Y. Kun and Z. Xiaojian, "Shunt APF control strategy on multiple synchronous rotating transform", Journal of Zhejiang University (Engineering Science), vol.47, no.4, (2013), pp.705-710.

[7] M. Liserre, R. Teodorescu and F. Blaabjerg, "Multiple harmonics control for three-phase grid converter systems with the use of PI-RES current controller in a rotating frame", IEEE Trans, Power Electron, vol.21, no.3, (2006), pp.836-841.

[8] Y. Ye, M. Kazerani and V. H. Quintana, "A novel modelling and control method for three-phase PWM converters. PESC, 32th Annual”, Vancouver, BC, Canada, (2001), pp.102-107.

[9] R. Grino, R. Cardoner and R. Costa-Castello, "Digital Repetitive Control of a Three-Phase Four-Wire Shunt Active Filter", Industrial Electronics, vol.54, no.3, (2007), pp.1495-1503.

[10] Y. Kun and C. Guozhu, "Compensation current control for DSTATCOM based on repetitive control. Automation of Electric Power Systems, vol.37, no.10, (2013), pp.80-85.

[11] M. Junxia, Z. Ziguan and L. Guanghui, "Detection of distorted current based on $i_{p}-i_{q}$ method and its physical realization", Power System Technology, vol.36, no.5, (2012), pp.125-131.

[12] S. Chi, W. Guanghui and B. Zengjun, "Detection for reactive and harmonic currents of unbalanced three-phase systems based on synchronous reference frame transformation", Proceedings of the CSEE. (2003).

[13] K. P. Sozański, "Sliding DFT control algorithm for three-phase active power filters. Applied Power Electronics Conference and Exposition, Twenty-First Annual IEEE”, (2006); Dallas TX. USA.

\section{Authors}

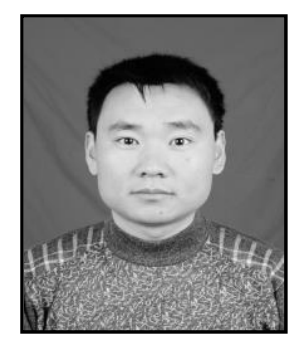

Xuehua Zhao, he is currently a student and also a $\mathrm{PhD}$ candidate at China University of Mining and Technology (CUMT), China. He received his MA Degree in Information and Electrical Engineering from CUMT in 2009. His research interests are the technologies of reactive compensation and harmonic restraint. Over the years, he has been actively involved in the development of the Cascade Multilevel Static Synchronous Compensator (STATCOM).

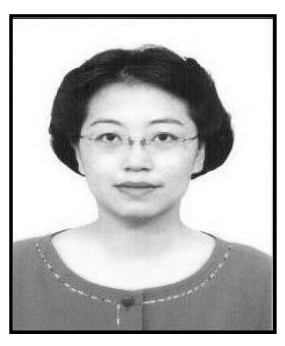

Liping Shi, she is born in 1964, Ph.D. She is a professor at School of Information and Electrical Engineering in CUMT. Her research interests are coal mine mechanical and electrical equipment and automation, application of power electronics in power systems, and equipment and power grid operation and fault diagnosis. She has published more than 30 research papers in journals and international conferences and she has won more than 10 the provincial scientific research awards. Now she is presiding research fund for the Doctoral Program of Higher Education of China under grant 20110095110014 and by the Key (Key grant) Project of Chinese Ministry of Education under grant 311021. 


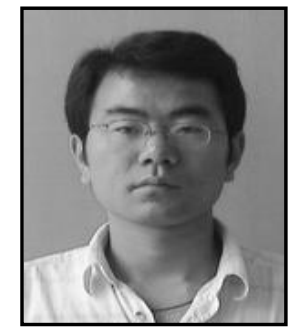

Zhenglong Xia, he is born in 1983, Ph.D. He is current a lecturer at School of electrical engineering \& automation, JIANGSU NORMAL UNIVERSITY, China. His research interests are reactive compensation of power system, fault diagnosis, distributed parallel processing, and neural network. 
International Journal of Control and Automation Vol. 8, No. 9 (2015) 